Carl Newell - Thomas Mullarkey • Mark Clyne

\title{
Radiation stress due to ocean waves and the resulting currents and set-up/set-down
}

The online version of the original article can be found at http:// dx. doi.org/10.1007/s10236-005-0009-2

C. Newell $(\bowtie) \cdot$ T. Mullarkey $\cdot$ M. Clyne Department of Civil Engineering,

National University of Ireland Galway,

University Road, Galway, Ireland

e-mail: carl.newell@nuigalway.ie

Tel.: +353-91-493086

Fax: $+353-91-750507$

\section{Ocean dynamics (2005) 55:499-514}

Unfortunately, Eqs. 12 and 13 were published with errors. The correct equations are given here.

$$
\begin{array}{r}
\frac{\partial U_{1}}{\partial t}+U_{1} \frac{\partial U_{1}}{\partial x}+g \frac{\partial \bar{\zeta}}{\partial x}+\frac{\overline{\tau_{1}^{B}}}{\rho(\bar{\zeta}+h)} \\
+\frac{1}{\rho(\bar{\zeta}+h)} \frac{\partial\left(S_{11}^{\prime}+S_{11}^{\prime \prime}\right)}{\partial x}=0 \\
\frac{\partial U_{2}}{\partial t}+U_{1} \frac{\partial U_{2}}{\partial x}-\frac{\overline{\tau_{2}^{B}}}{\rho(\bar{\zeta}+h)} \\
+\frac{1}{\rho(\bar{\zeta}+h)} \frac{\partial\left(S_{12}^{\prime}+S_{12}^{\prime \prime}\right)}{\partial x}=0
\end{array}
$$

